\title{
Erratum: Unified solution of the inverse capacity problem \\ [Phys. Rev. E 57, 1302 (1998)]
}

Chen Nan-xian* and Rong Er-qian

\section{[S1063-651X(98)09405-9]}

PACS number(s): 05.90. $+\mathrm{m}, 65.40 .+\mathrm{g}$, 02.10.Lh, 02.30.Sa, 99.10. $+\mathrm{g}$

Equation (20) should read

$$
g(\nu)=\frac{2}{r k \nu} \sum_{m=2}^{\infty} \frac{a_{2 m-1}(h \nu / k)^{2 m-1}}{(-1)^{m+1}(2 \pi)^{2 m} B_{2 m}}
$$

Equation (21) should read

$$
g(\nu)=\frac{2}{r k \nu} \frac{(-1)^{2+1} a_{3}(h \nu / k)^{3}}{(2 \pi)^{4} B_{4}}=\frac{2}{r k \nu} \frac{(-1)^{2+1} a_{3}(h \nu / k)^{3}}{(2 \pi)^{4}(-1 / 30)}=\frac{15 a_{3} h^{3}}{4 \pi^{4} k^{4} r} \nu^{2} .
$$

The deduction of (B13) and (B14) should be

$$
\begin{aligned}
1=\frac{-\Gamma(z)}{2 \pi i} \int_{\infty}^{(0+)} e^{-t}(-t)^{-z} d t & =\frac{-\Gamma(m+1)}{2 \pi i} \int_{\infty}^{(0+)} e^{-(n+1) x}[-x(n+1)]^{-(m+1)}(n+1) d x \\
& =\frac{-m !}{2 \pi i} \int_{\infty}^{(0+)} e^{-(n+1) x}(n+1)^{-m}(-x)^{-(m+1)} d x . \\
& \rightarrow(n+1)^{m}=\frac{-m !}{2 \pi i} \int_{\infty}^{(0+)} e^{-(n+1) x}(-x)^{-(m+1)} d x .
\end{aligned}
$$

Then,

$$
\begin{aligned}
\lim _{0<y \rightarrow 1^{-1}} \sum_{n=0}^{\infty}(n+1)^{m} y^{n+1} & =\frac{-m !}{2 \pi i} \int_{\infty}^{(0+)}(-x)^{-(m+1)} \sum_{n=0}^{\infty} e^{-(n+1) x} d x \\
& =\frac{-m !}{2 \pi i} \int_{\infty}^{(0+)}(-x)^{-(m+1)} e^{-x} \sum_{n=0}^{\infty} e^{-n x} d x \\
& =\frac{-m !}{2 \pi i} \int_{\infty}^{(0+)}(-x)^{-(m+1)} \frac{e^{-x}}{1-e^{-x}} d x .
\end{aligned}
$$

The deduction of (B19) should read as follows.

The integration in right-hand side has a pole of rank $(m+2)$ at $x=0$, thus

$$
\begin{aligned}
\frac{1}{2 \pi i} \int_{\infty}^{(0+)}(-x)^{-(m+1)} \frac{e^{-x}}{1-e^{-x}} d x & =\operatorname{Res}_{x=0}\left[(-x)^{-(m+1)} \frac{e^{-x}}{1-e^{-x}}\right] \\
& =\operatorname{Res}_{x=0}\left[(-x)^{-(m+1)} \frac{1}{e^{x}-1}\right] \\
& =\frac{(-1)^{m+1}}{(m+1) !} \lim _{x \rightarrow 0} \frac{\partial^{(m+1)}}{\partial x^{(m+1)}}\left[\frac{x^{m+2} x^{-(m+1)}}{e^{x}-1}\right] \\
& =\frac{(-1)^{m+1}}{(m+1) !} \lim _{x \rightarrow 0} \frac{\partial^{(m+1)}}{\partial x^{(m+1)}}\left[\frac{x}{e^{x}-1}\right] .
\end{aligned}
$$

\footnotetext{
*Author to whom correspondence should be addressed. Address correspondence to Institute of Applied Physics, Beijing University of Science and Technology, Beijing 100083, China. 
Based on the generating function of Bernoulli numbers $B_{n}$

$$
\frac{t}{e^{t}-1}=\sum_{n=0}^{\infty} \frac{t^{n}}{n !} B_{n}
$$

we have

$$
\begin{aligned}
\mathrm{B}(16) & =\left.\frac{(-1)^{m+1}}{(m+1) !} \frac{\partial^{(m+1)}}{\partial x^{(m+1)}}\left[\sum_{n=0}^{\infty} \frac{x^{n}}{n !} B_{n}\right]\right|_{x=0} \\
& =\frac{(-1)^{m+1}}{(m+1) !} \frac{\partial^{(m+1)}}{\partial x^{(m+1)}}\left[\frac{x^{m+1}}{(m+1) !} B_{m+1}\right]=\frac{(-1)^{m+1}}{(m+1) !}\left[\frac{(m+1) !}{(m+1) !} B_{m+1}\right]=(-1)^{m+1}\left[\frac{B_{m+1}}{(m+1) !}\right] .
\end{aligned}
$$

Therefore, it is given that

$$
\begin{aligned}
\lim _{0<x \rightarrow 1} \sum_{n=0}^{\infty}(n+1)^{m} x^{n+1} & =-\frac{m !}{2 \pi i} \int_{\infty}^{\left(0^{+}\right)}(-x)^{-(m+1)} \frac{e^{-x}}{1-e^{-x}} d x \\
& =-m !\left[\frac{1}{2 \pi i} \int_{\infty}^{\left(0^{+}\right)}(-x)^{-(m+1)} \frac{e^{-x}}{1-e^{-x}} d x\right]=(-m !)\left[(-1)^{m+1} \frac{B_{m+1}}{(m+1) !}\right]=\frac{(-1)^{m} B_{m+1}}{m+1} .
\end{aligned}
$$

Therefore, for any natural number $m$,

$$
\lim _{0<x \rightarrow 1^{-}} \sum_{n=0}^{\infty}(n+1)^{m} x^{n+1}=\lim _{0<x \rightarrow 1^{-}} \sum_{n=1}^{\infty} n^{m} x^{n}=(-1)^{m} \frac{B_{m+1}}{m+1}= \begin{cases}0, & m=2 k>0 \\ -\frac{B_{2 k}}{2 k}=\zeta(1-2 k) & m=2 k-1>0 .\end{cases}
$$

Notice that $B_{2 k-1}=0$. Thus the first theorem is proved as

$$
\lim _{0<x \rightarrow 1^{-}} \sum_{n=1}^{\infty} n^{m} x^{n}= \begin{cases}0, & m=2 k>0, \\ \zeta(1-2 k), & m=2 k-1>0 .\end{cases}
$$

